\title{
Effet de l'hypoxie sur les concentrations plasmatiques de gastrine et de polypeptide inhibiteur gastrique (GIP) chez le veau nouveau-né
}

\author{
A Mouats ${ }^{1}$, P Guilloteau', JA Chayvialle², R Toullec1, \\ C Bernard2, JF Grongnet ${ }^{1}$, GT Dos Santos ${ }^{1}$ \\ avec la collaboration technique de M Beaufils, J Lareynie \\ 1 Laboratoire du Jeune Ruminant, INRA, 65, rue de Saint-Brieuc, 35042 Rennes Cedex; \\ 2 INSERM U55, 69374 Lyon Cedex, France
}

\begin{abstract}
Summary - Calves were subjected to experimental hypoxia from 0.5 to $4 \mathrm{~h}$ after birth. The plasma level of immunoreactive gastric inhibitory polypeptide was higher $(P<0.05)$ than in control calves during hypoxia and 2 and $7 \mathrm{~h}$ later. However, the gastrin level was not lower during treatment and was higher ( $\mathrm{P}<0.05) 3,6$ and $13 \mathrm{~h}$ later. Hypoxia could have changed circulating levels or degradation rates of these peptides and could have delayed abomasal emptying.
\end{abstract}

Introduction - Lors de la naissance du jeune mammifère, la fonction de digestion doit devenir rapidement efficace. Chez le bébé, les mécanismes hormonaux intervenant dans les adaptations digestives peuvent être perturbés en cas d'hypoxie (Lucas et al, 1979). Le but de ce travail est d'étudier l'influence de l'hypoxie sur l'évolution des taux circulants des hormones digestives chez le veau nouveau-né. Nous rapportons ici les résultats concernant la gastrine et le GIP dont les taux ont été particulièrement modifiés.

Matériel et Méthodes - Dix huit veaux PieNoirs sont répartis à la naissance en deux lots de 9 animaux ( $T$ témoins et $H$ hypoxiés, pesant respectivement $44,0 \pm 2,4$ el $45,4 \pm 2,3 \mathrm{~kg}$ à la naissance (moyennes \pm écarts types des moyennes). Les veaux $H$ sont soumis à une hypoxie expérimentale (inhalation d'un mélange comprenant $8,5 \%$ d'oxygène et $91,5 \%$ d'azote) depuis $0 \mathrm{~h} 30$ jusqu'à $4 \mathrm{~h}$ après la naissance. Trois repas de colostrum sont distribués au seau à tétine 6,13 et $25 \mathrm{~h}$ après la naissance, à raison de $25 \mathrm{~g} / \mathrm{kg}$ de poids vif. Des prises de sang sont effectuées dans la veine jugulaire (fig 1) comme indiqué précédemment et les plasmas sont conservés à $-45^{\circ} \mathrm{C}$ jusqu'aux analyses (Guilloteau ot al, 1986).

Résultats et Discussion - Chez les veaux $T$, les taux plasmatiques de GIP et de gastrine immunoréactifs diminuent depuis la naissance jusqu'à l'ingestion du premier repas de colostrum puis augmentent (fig 1). Cependant, seule l'évolution observée de la naissance à $6 \mathrm{~h}$ pour la gastrine est significative. Chez les veaux $H$, le taux de GIP reste voisin de celui observé chez les veaux T pendant les 90 premières minutes d'hypoxie mais est 2,5 fois plus élevé $1 \mathrm{~h}$ plus tard $(P<0,05)$; après l'arrêt du traitement, la différence met plus de $3 \mathrm{~h}$ pour disparaître définitivement. Dans l'espèce humaine, une naissance dystocique provoque également un accroissement du taux circulant de GIP (Lucas et al, 1979). Le taux de gastrine est voisin dans les deux lots pendant l'hypoxie mais, dès la fin du traitement, il de- 

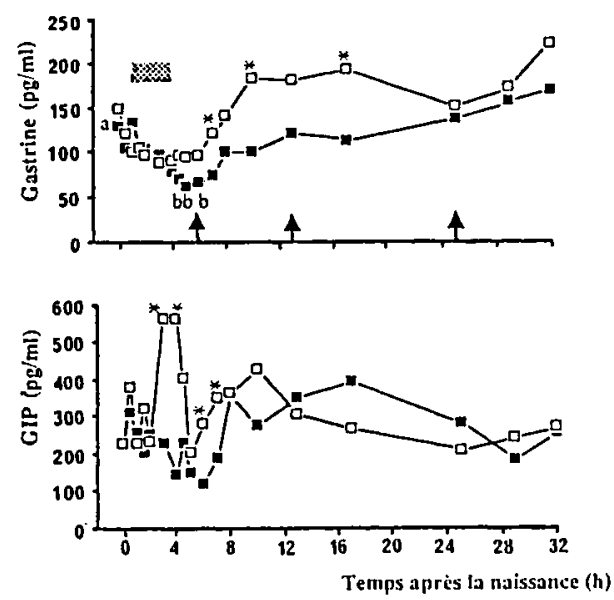

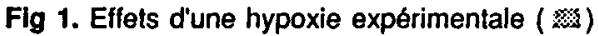
sur l'évolution des taux plasmatiques de gastrine et de GIP immunoréactifs chez le veau nouveau-né receivant 3 repas de colostrum ( 4 ). $\mathbf{m}$ : témoins, $\square$ : hypoxiés; * : différences significatives entre lots $(P \leq 0,05)$; $\mathrm{a}, \mathrm{b}$ : dans le lot témoin, les valeurs accompagnées de lettres différentes sont significativement différentes $(P \leq 0,05)$.

vient plus élevé dans le lot $\mathrm{H}$ et lé reste pendant $13 \mathrm{~h}(P<0,05$ après 3,6 et $13 \mathrm{~h})$. Le taux plasmatique de glucose est beaucoup plus élevé dans le lot $\mathrm{H}$ pendant l'hypoxie $(2,2 \pm 0,2 \mathrm{~g} / \mathrm{l}$ au lieu de $1,2 \pm 0,3$ après 1 h $30 ; P<0,05$ ), ce qui, avec l'accroissement considérable du taux de GIP, devrait entraîner une réduction de la libération de gastrine (Wiener et al, 1987). Cependant, les taux circulants de gastrine et de GIP sont les résultantes de plusieurs phénomènes (libération, captation et catabolisme) qui pourraient être diversement affectés par la réduction de la température corporelle $\left(-3^{\circ} \mathrm{C}\right.$ à la fin du traitement). Celle-ci revient progressivement à sa valeur normale au cours des 4 premières heures qui suivent. En revanche, le taux plasmatique de glucose diminue plus fortement jusqu'à la prise du premier repas et demeure très bas pendant les 8 premières heures postprandiales dans le lot $\mathrm{H}$. Pendant le mème temps, dans le lot $T$, il augmente et devient plus élevé que dans le lot $H(1,1 \pm 0,1 \mathrm{~g} / \mathrm{l}$ au lieu de $0,5 \pm 0,11 \mathrm{~h}$ après le repas; $P<0,05)$. L'évacuation gastrique pourrait donc être ralentie, ce qui provoquerait un maintien prolongé de la distension de la caillette contribuant à accroître la libération de gastrine (Wiener et al, 1987).

En conclusion, l'hypoxie expérimentale appliquée à la naissance provoque des perturbations métaboliques qui agissent sur le fonctionnement du tube digestif du veau et sur les taux circulants de certains peptides régulateurs.

Guilloteau P, Corring T, Chayvialle JA, Bernard C, Sissons JW, Toullec R (1986) Reprod Nutr Dév 26, 717-728

Lucas A, Sarson DL, Adrian TE, Aynsley-Green A, Bloom SR (1979) Lancet ii, 968

Wiener I, Khalil T, Thompson JC, Greeley GH, Rayford PL (1987) in: Gastrointestinal Endocrinology (Thompson JC, Greeley GH, Rayford PL, Townsend CM, eds) McGraw-Hill Book Company, New York, 194-212 